\title{
ORIGINAL
}

\section{Falsely elevated thyroid hormone levels caused by anti- ruthenium interference in the Elecsys assay resembling the syndrome of inappropriate secretion of thyrotropin}

\author{
Kenji Ohba ${ }^{1)}$, Jaeduk Yoshimura Noh${ }^{2)}$, Tsuyoshi Unno ${ }^{3)}$, Tomoaki Satoh ${ }^{3)}$, Kunihiro Iwahara ${ }^{4)}$, \\ Akio Matsushita $^{1)}$, Shigekazu Sasaki ${ }^{1)}$, Yutaka $\mathrm{Oki}^{1)}$ and Hirotoshi Nakamura ${ }^{1)}$ \\ 1) Second Division, Department of Internal Medicine, Hamamatsu University School of Medicine, Hamamatsu 431-3192, Japan \\ 2) Ito Hospital, Tokyo 150-8308, Japan \\ 3) IM\&CC Life Cycle Management, IVD, Roche Diagnostics K.K., Tokyo 105-0014, Japan \\ 4) Department of Laboratory Medicine, Hamamatsu University School of Medicine, Hamamatsu 431-3192, Japan
}

\begin{abstract}
The syndrome of inappropriate secretion of thyrotropin (SITSH) is defined as the inappropriate non-suppression of serum TSH in the presence of elevated free thyroid hormone; TSH-secreting pituitary adenomas and the syndrome of resistance to thyroid hormone are the main etiologies of SITSH. In addition, erroneous thyroid function testing may result in the diagnosis of this syndrome. A 63-year-old woman was referred because of suspected SITSH. Laboratory tests showed a normal TSH (0.52 $\mu \mathrm{IU} / \mathrm{L}$; normal range: 0.5-5.0) measured by sandwich Elecsys, and elevated FT4 (3.8 ng/dL; normal range: $0.9-1.6)$ and FT3 (7.6 pg/mL; normal range: $2.3-4.0)$, determined by competitive Elecsys. To exclude possible assay interference, aliquots of the original samples were retested using a different method (ADVIA Centaur), which showed normal FT4 and FT3 levels. Eight hormone levels, other than thyroid function tests measured by competitive or sandwich Elecsys, were higher or lower than levels determined by an alternative analysis. Subsequent examinations, including gel filtration chromatography, suggested interference by substances against ruthenium, which reduced the excitation of ruthenium, and resulted in erroneous results. The frequency of similar cases, where the FT4 was higher than $3.2 \mathrm{ng} / \mathrm{dL}$, in spite of a non-suppressed TSH, was examined; none of 10 such subjects appeared to have method-specific interference. Here, a patient with anti-ruthenium interference, whose initial thyroid function tests were consistent with SITSH, is presented. This type of interference should be considered when thyroid function is measured using the Elecsys technique, although the frequency of such findings is likely very low.
\end{abstract}

Key words: Anti-ruthenium interference, Electrochemiluminescent immunoassay (ECLIA), Elecsys, Eclusys, Syndrome of inappropriate secretion of thyrotropin

THE SYNDROME of inappropriate secretion of thyrotropin (SITSH) is a clinical condition defined as inappropriate non-suppression of serum TSH despite elevated free thyroid hormone; this condition is caused by TSH-producing pituitary adenomas (TSHomas), the syndrome of resistance to thyroid hormone $(\mathrm{RTH})$ and other rare disorders [1-4]. The laboratory assays of TSH, FT4 and FT3 are continuously being improved. However, some cases have been reported in which erroneous thyroid function testing was noted due to methodological interference, such as anti-iodothyro-

Submitted Feb. 29, 2012; Accepted Apr. 16, 2012 as EJ12-0089 Released online in J-STAGE as advance publication May 11, 2012 Correspondence to: Kenji Ohba, Hamamatsu University School of Medicine, 1-20-1 Handayama, Higashi-ku, Hamamatsu 431-3192, Japan. E-mail: kenji26@hama-med.ac.jp nine antibodies, anti-TSH antibodies or heterophile antibodies, which resemble SITSH [5]. Failure to recognize such laboratory errors can lead to undesirable consequences, such as unnecessary patient examinations (e.g. magnetic resonance imaging or genetic analysis of the thyroid hormone receptor $\beta$ gene) and/ or improper treatment (e.g. pituitary surgery or thyroidectomy). Here, a patient with interfering substances against ruthenium whose initial thyroid function tests were consistent with SITSH is reported.

\section{Case Study}

A 63-year-old woman presented to a community hospital with a diffuse goiter in 2004 . The past med- 
ical history was significant for rheumatoid arthritis. Laboratory tests showed mild SITSH; a normal TSH (0.52 $\mu \mathrm{IU} / \mathrm{mL}$; normal range: 0.5-5.0) measured by sandwich Elecsys (Roche Diagnostics K.K., Tokyo, Japan), and elevated FT4 (3.8 ng/dL; normal range: 0.9-1.6) and FT3 levels (7.6 pg/mL; normal range: $2.3-$ 4.0) determined by competitive Elecsys. After treatment with methimazole, the patient complained of general malaise and deterioration of the goiter. Transsphenoidal surgery was recommended for a suspected TSHoma; however, the patient declined and was referred to this hospital in 2009. The methimazole was discontinued as the serum showed a normal FT4 (1.1 ng/dL), but very high TSH $(19.1 \mu \mathrm{IU} / \mathrm{L})$. The symptoms improved one month later; however, the FT4 and FT3 increased to $3.9 \mathrm{ng} / \mathrm{dL}$ and $7.4 \mathrm{pg} / \mathrm{mL}$, despite the normal TSH (Table 1). The patient was admitted to the hospital for further evaluation.

A physical examination showed that the body temperature was $36.6^{\circ} \mathrm{C}$, blood pressure was $133 / 69 \mathrm{mmHg}$ and the heart rate was 76 beats $/ \mathrm{min}$. The patient measured $150 \mathrm{~cm}$ in height and weighed $49.0 \mathrm{~kg}$. Palpation revealed a mildly enlarged diffuse goiter. Mild anemia and slightly elevated hepatic transaminase levels were detected on the routine blood chemistries. Ultrasound showed a diffuse hypoechoic and moderately heterogeneous thyroid gland. Anti-thyroperoxidase antibodies were positive with a titer as low as $0.4 \mathrm{U} / \mathrm{mL}$ (normal range: $<0.3 \mathrm{U} / \mathrm{mL}$; Cosmic Co., Tokyo, Japan). The TSH receptor antibodies and anti-thyroglobulin antibodies were both negative.

For the evaluation of SITSH, magnetic resonance imaging was performed; there was no pituitary adenoma detected. Genetic analysis of the thyroid hormone receptor $\beta$ gene was conducted after providing genetic counseling and obtaining written informed consent, as described previously [4]; no mutations were identified. The institutional review board approved the study protocol. To exclude method-specific interference, the thyroid function tests were performed again using the ADVIA Centaur (Siemens, Munich, Germany) chemiluminescence assay (CLIA). The results were normal (Table 1). Of note, four hormone levels, other than thyroid, measured by competitive Elecsys, were higher than those hormones measured by ruthenium-independent ADVIA Centaur. In addition, four other hormone levels, measured by sandwich Elecsys, were lower than the hormones measured by an alternative method (Table 1).

To clarify the mechanism underlying the falsely ele- vated or decreased hormone levels, subsequent examinations were performed after obtaining written informed consent. First, the patient's thyroid function tests were assayed using a modified research reagent, ruthenium was replaced by sulfonated ruthenium to reduce the effect of interfering substances, because possible interference of ruthenium or streptavidin, common components of Elecsys, was considered. As shown in Table 2, the results showed a marked reduction of FT4 and FT3. Second, the patient's serum was measured after precipitation using streptavidin beads. The results showed no significant reduction in TSH, FT4 and FT3, which eliminated the possibility of interference with streptavidin. Third, the patient's serum was fractionated by HPLC gel filtration and thyroid function tests were measured in each fraction, as described previously [4]. When control serum was fractionated, FT4 eluted at one peak, with a molecular weight between IgG and $\mathrm{TSH}$; this likely represented FT4 in equilibrium with T4 bound to plasma binding proteins. By contrast, FT3 did not elute at a clear peak but rather as an elevation of the baseline (Fig. 1-A). In the case of the patient's

Table 1 Hormone levels measured with different methods

\begin{tabular}{lccc}
\hline \multicolumn{3}{l}{ Variable } & \multicolumn{2}{l}{ Elecsys } & ADVIA Centaur \\
\hline \multicolumn{2}{l}{ Competitive method } \\
FT4 & $\mathrm{ng} / \mathrm{dL}$ & $3.9(0.9-1.6)$ & $1.3(0.8-1.9)$ \\
FT3 & $\mathrm{pg} / \mathrm{mL}$ & $7.4(2.3-4.0)$ & $3.1(2.2-4.1)$ \\
Testosterone & $\mathrm{ng} / \mathrm{mL}$ & $108.8(13-69)^{*}$ & $16.0(3-67)^{*}$ \\
Estradiol & $\mathrm{pg} / \mathrm{mL}$ & $362.7(<21)^{*}$ & $12.9(<39)^{*}$ \\
Progesterone & $\mathrm{ng} / \mathrm{mL}$ & $1.4(<0.4)^{*}$ & $0.2(<0.9)^{*}$ \\
Cortisol & $\mu \mathrm{g} / \mathrm{dL}$ & $29.2(6.2-19.4)$ & $7.7(4.5-21.1)$ \\
Sandwich method & & \\
TSH & $\mu \mathrm{IU} / \mathrm{mL}$ & $0.57(0.5-5.0)$ & $1.10(0.4-4.0)$ \\
LH & $\mathrm{mIU} / \mathrm{mL}$ & $22.6(5.7-64.3)^{*}$ & $36.9(13.3-61.6)^{*}$ \\
FSH & $\mathrm{mIU} / \mathrm{mL}$ & $40.0(<157.8)^{*}$ & $61.2(<142.8)^{*}$ \\
PRL & $\mathrm{ng} / \mathrm{mL}$ & $4.4(6.1-30.5)$ & $6.5(0.6-18.5)$ \\
Insulin & $\mu \mathrm{U} / \mathrm{mL}$ & $10.1(1.8-12.2)$ & $15.2(2.2-12.4)$ \\
\hline
\end{tabular}

Parentheses show the normal ranges. *Normal range for postmenopausal women.

Table 2 Thyroid hormone levels determined by the modified research reagent

\begin{tabular}{cccccc}
\hline \multirow{2}{*}{ Specimin } & \multicolumn{2}{c}{ FT4 $(\mathrm{ng} / \mathrm{dL})$} & & \multicolumn{2}{c}{ FT3 $(\mathrm{pg} / \mathrm{mL})$} \\
\cline { 2 - 3 } \cline { 5 - 6 } & Elecsys & $\begin{array}{c}\text { Modified } \\
\text { research reagent }\end{array}$ & & Elecsys & $\begin{array}{c}\text { Modified } \\
\text { research reagent }\end{array}$ \\
\hline Patient & 7.77 & 1.60 & & 9.57 & 3.80 \\
Control & 1.24 & 1.35 & & 3.01 & 3.03 \\
\hline
\end{tabular}


A
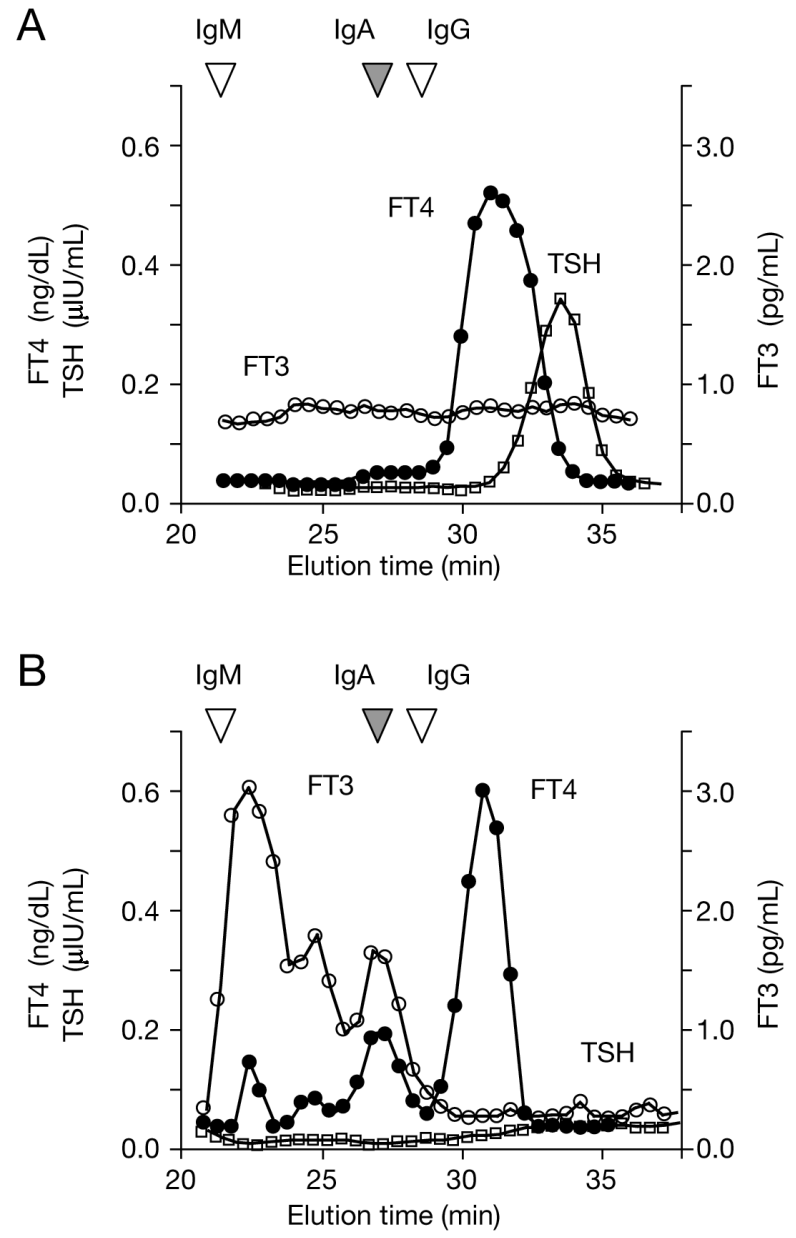

C

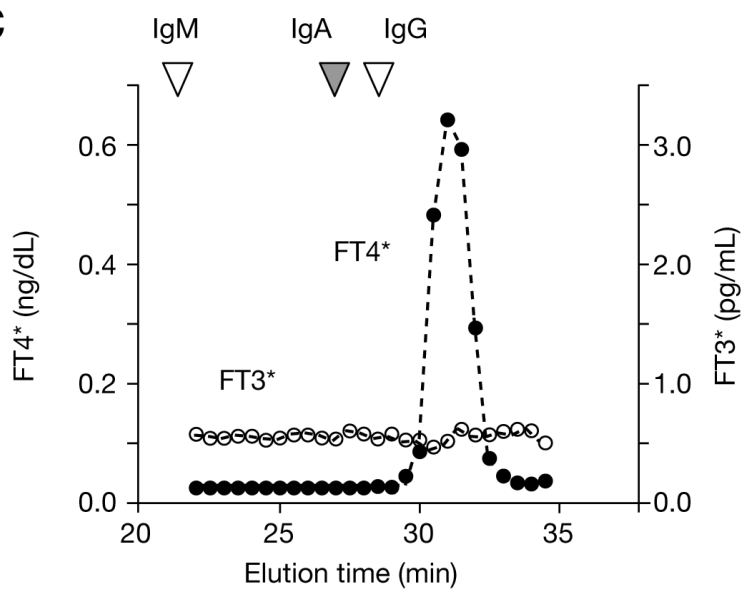

Fig. 1 Gel filtration chromatography of a serum sample from a control subject $(\mathrm{A})$ and the patient $(\mathrm{B}, \mathrm{C})$

The peak fractions of $\operatorname{IgM}, \operatorname{IgA}$ and $\operatorname{IgG}$ are shown as markers of molecular mass. The solid circle, open square and open circle indicate FT4, TSH and FT3, respectively. *Determined by the modified research reagent. serum, three additional peaks of FT4 and FT3, but no TSH peak, were observed (Fig. 1-B). Additional peaks of thyroid hormone with a molecular weight between IgM and IgG disappeared when each fraction was measured with the modified research reagent (Fig. 1-C). These results suggested that several substances interfered with ruthenium, which resulted in the elevation of thyroid hormone. Although the retention time of one of the common peaks of FT4 and FT3 was the same as that of IgA, at $27.0 \mathrm{~min}$, only a minor part of these hormones eluted in this fraction. This excluded IgAtype antibodies from playing a major role in the interference with ruthenium. Alternately, one of the interfering substances might have had a molecular weight almost equal to that of IgA.

Finally, to evaluate the frequency of similar cases, 10 subjects were recruited with serum FT4 measured by Elecsys higher than $3.2 \mathrm{ng} / \mathrm{dL}$ and TSH levels not lower than $0.5 \mu \mathrm{IU} / \mathrm{L}$, among the patients presented to Ito Hospital between January 2004 and January 2011. The institutional review board approved this study. The results showed that none of the 10 subjects had anti-ruthenium interference; all of the stored serum samples had elevated FT4 determined by ruthenium-independent CLIA using Lumipulse f (Fuji Rebio Inc., Tokyo, Japan). Further examinations were not conducted in any of these additional cases.

\section{Discussion}

TSH-oma and RTH are the two main causes of SITSH; other causes, such as an ectopic TSH-producing tumor or method-specific interference, are very rare [1-4]. The patient described here, was referred to us because of suspected SITSH. Further evaluation showed interfering substances against ruthenium in the Elecsys analysis. Elecsys, referred to as Eclusys in Japan, is an electrochemiluminescent immunoassay (ECLIA) involving ruthenium as the luminescent material [6]. Although this fully automated rapid assay provides prompt diagnosis of patients with thyroid disease, and has about $25 \%$ of the market share in Japan (Roche Diagnostics, personal communication, November 27, 2011), method-specific interference associated with ruthenium or streptavidin has been described previously [7-10]. After Roche Diagnostics introduced improved reagents for Elecsys, in the summer of 2006, Ando and colleagues first reported antiruthenium interference in competitive Elecsys assays 
for FT3 and FT4 [7]. Subsequently, similar interference, for competitive total T4 and sandwich TSH was confirmed by Mandel et al. [9] and Buijis et al. [10].

Elecsys methods are classified as competitive and non-competitive sandwich assays. For the competitive assay, the amount of emission produced by the excited ruthenium is inversely proportional to the concentration of the hormones present. By contrast, in the sandwich assay, the amount of emission correlates positively with the hormone levels $[7,11]$. Therefore, an interfering substance reduces the amount of emission produced by the excited ruthenium, resulting in falsely elevated or depressed hormone levels in the competitive or sandwich Elecsys assays [10]; a detailed description of the mechanisms involved has not been reported. The main unresolved question is why this type of interference is found only in thyroid function tests, when interfering substances could theoretically affect all Elecsys analyses.

This is the first case reported showing anti-ruthenium interference in hormone assays other than thyroid function tests. The findings reported here, make it easier to understand a previously proposed mechanism. The patient reported might have had higher serum levels of the interfering substance than previous patients, making it easier to detect than in prior cases, and suggesting a wider effect on a variety of Elecsys analyses. In the patient reported here, the interference in the competitive Elecsys appeared to be more significant than in the sandwich assay (Table 1). This finding supports the hypothesis, since competitive Elecsys assays involve less ruthenium than the sandwich assay (Roche Diagnostics, personal communication, November 27, 2011). This type of interference may have been missed in earlier studies. For example, Heijboer et al. reported that no interference was observed in the Elecsys TSH assay, although TSH measured by Elecsys in one of their patients was apparently as low as $0.32 \mathrm{mU} / \mathrm{L}$ (normal range: 0.3-4.5) compared to the level determined by an alternative method, which was $13.0 \mathrm{mU} / \mathrm{L}$ (normal range: 0.5-5.0) [8]. Similarly, Buijs et al. found lower AFP levels measured by Elecsys than with an alternative method; however, they reported that the Elecsys AFP assay did not show any clear sign of interference [10]. In a recent case study of a patient with suspected vitamin D toxicity, Khieng et al. reported interference associated with falsely high results of competitive Elecsys (i.e. vitamin D and FT4) and low results in the sandwich assay (i.e. PTH and TSH) [11]. Although the interfering factor could not be identified, anti-ruthenium interference may have accounted for the erroneous results in their patient.

Little information is available on substance interference with ruthenium, although anti-ruthenium antibodies have been considered (Roche Diagnostics, personal communication, November 27, 2011). Ruthenium is a rare transition metal belonging to the platinum group noted in the periodic table; it is mostly used for wearresistant electrical contacts and the production of thickfilm resistors. Bujis et al. proposed that anti-ruthenium antibodies might be induced by the intake of ruthenium in the food chain or exposure to residues on clothing [10]. For the patient reported here, the possible involvement of antibodies does not seem likely (Fig. 1), and the specific interfering substances could not be identified.

The prevalence of "anti-ruthenium interference" remains unknown. As SITSH due to TSHoma was initially considered in this patient, it was decided to evaluate its frequency in subjects with elevated FT4 and measurable TSH. Since transient minimum hyperthyroxinemia with detectable TSH is often caused by unstable thyroid disease on treatment and since it was reported that the mean serum FT4 was $42.0 \mathrm{pmol} / \mathrm{L}(3.3 \mathrm{ng} / \mathrm{dL})$ in 18 patients with TSHoma [2], the $3.2 \mathrm{ng} / \mathrm{dL}$ (i.e. 2-fold the upper limit) was interpreted as an elevated FT4. A similar interference in 10 such subjects could not be detected. A marked interference in Elecsys FT4 assays appears to be rare; however, a more mild interference might be more frequent than generally considered.

In conclusion, the mechanism associated with antiruthenium interference was examined in a patient that had elevated thyroid hormone levels due to this interference. Although similar cases appear to be rare, the case reported here raises the possibility that laboratory artifact should be considered and excluded in patients with suspected SITSH when thyroid function tests are measured by Elecsys assays.

\section{Conflict of Interest}

All authors have nothing to declare.

\section{Funding}

This work was supported, in part, by a Health Sciences Research Grant and a Grant-in Aid for Scientific Research to H Nakamura from the Ministry of Education, Culture, Sports, Science and Technology in Japan. 


\section{References}

1. Weintraub BD, Gershengorn MC, Kourides IA, Fein H (1981) Inappropriate secretion of thyroid-stimulating hormone. Ann Intern Med 95: 339-351.

2. Beck-peccoz P, Persani L (2005) Thyrotropin-induced thyrotoxicosis. In: Braverman LE, Utiger RD (eds) Werner and Ingbar's the Thyroid. A Fundamental and Clinical Text, 9th edition. Lippincott Williams \& Wilkins, Philadelphia: 501-507.

3. Refetoff S, Dumitrescu AM (2007) Syndromes of reduced sensitivity to thyroid hormone: genetic defects in hormone receptors, cell transporters and deiodination. Best Pract Res Clin Endocrinol Metab 21: $277-$ 305.

4. Ohba K, Shirakawa K, Okawa Y, Iwaki H, Matsunaga H, et al. (2011) Syndrome of inappropriate secretion of thyrotropin associated with thymoma-related peripheral nerve hyperexcitability. Endocr J 58: 597-602.

5. Despres N, Grant AM (1998) Antibody interference in thyroid assays: a potential for clinical misinformation. Clin Chem 44: 440-454.

6. Kulmala S, Suomi J (2003) Current status of modern analytical luminescence methods. Analytica Chimica Acta 500: 21-69.

7. Ando T, Yasui J, Inokuchi N, Usa T, Ashizawa K, et al. (2007) Non-specific activities against ruthenium crosslinker as a new cause of assay interference in an electrochemilluminescent immunoassay. Intern Med 46: 12251229.

8. Heijboer AC, Ijzerman RG, Bouman AA, Blankenstein MA (2009) Two cases of anti-ruthenium antibody interference in Modular free thyroxine assay. Ann Clin Biochem 46: 263-264.

9. Mandal S, Kin AJ, Lee HK, Brough T, Yeo KTJ (2009) Discordant Roche Elecsys Thyroid Function Tests due to Interference From Antibodies Against RutheniumTag and Streptavidin. Program of 2009 ASCP Annual Meeting, 60 (Abstract).

10. Buijs MM, Gorgels JP, Endert E (2011) Interference by anti-ruthenium antibodies in the Roche thyroid-stimulating hormone assay. Ann Clin Biochem 48: 276-281.

11. Khieng V, Stevens C (2010) Vitamin D Toxicity?: A Case Study. N Z J Med Lab Sci 64: 44-50. 